PESQUIMAT, Revista de la Fac.CC.MM. de la UNIVERSIDAD NACIONAL MAYOR DE SAN MARCOS

Vol.IV, $N^{\circ} 1$,pag.39-50,LIMA-PERÚ. Julio 2001

\title{
GLOBAL EXISTENCE OF SOLUTIONS FOR THE DEGENERATE WAVE EQUATIONS OF KIRCHOFF TYPE WITH NONLINEAR DISSIPATIVE TERM OF VARIABLE COEFFICIENT
}

\author{
E. Cabanillas, J. Bernui \& Z. Huaringa ${ }^{1}$
}

\begin{abstract}
In this paper we investigate the global existence and decay of solutions to a degenerate wave equations with nonlinear dissipative term of variable coefficient.
\end{abstract}

\section{INTRODUCTION}

The objective of this paper is to study the global existence and the decay property of the nonlinear system:

$(P) \mid \begin{array}{ll}u^{\prime \prime}-M\left(\int_{\Omega}|\nabla u|^{2} d x\right) \triangle u+a(x) g\left(u^{\prime}\right)=0 & \text { in } \quad Q=\Omega \times] 0, T[ \\ u=0 & \left.\text { in } \quad \sum=\Gamma \times\right] 0, T[ \\ u(x, 0)=u_{0}(x), \quad \frac{\partial u}{\partial t}(x, 0)=u_{1}(x) & \text { in } \Omega\end{array}$

where $\Omega$ is a bounded open domain in $\mathbb{R}^{N}(N \geq 1)$ with a smooth boundary $\Gamma, T>0, M(s)=s, \forall s \geq 0, \Delta$ is the Laplace operator, $g$ and $a$ are functions satisfying suitables conditions.

Existence of global solutions to the system $(P)$ has been investigated by many authors (ef. [1], [2], [4], [7], [8], etc) for different and positive constant, with a positive or non-negative function. Mochizuki [5] investigated the nondegenerate problem con dissipative term $a(x, t) u^{\prime}$. Our purpose in this time is to prove the global existence and decay rate of solution for the case: $M(s)=s$ and $a(x)$ is a positive function.

\footnotetext{
${ }^{1}$ Universidad Nacional Mayor de San Marcos. Facultad de Ciencias Matemáticas.
} 


\section{PRELIMINARIES}

In the sequel $L^{p}(\Omega), 1 \leq p<\infty$ will denote the collection of $L$ functions which are pth-integrable over $\Omega$. For $m \in \mathbb{N}$, the space $H^{m}(\Omega)$ is the Sobolev class of the functions of the spatial variable $x$ wich along with their first $m$ derivates belong to $L^{2}(\Omega)$ (See, for example Medeiros \& Milla Miranda [3]) and the closure in $H^{m}(\Omega)$ of the space $D(\Omega)$ of the test functions on $\Omega$ is denoted by $H_{0}^{m}(\Omega)$ the inner product and norm of $L^{2}(\Omega)$ are represented by $(\cdot, \cdot)$ and $|\cdot|$ respectively.

Let $X$ be a Banach space, $T>0$ or $T=+\infty$ and $1 \leq p \leq \propto$. denote by $L^{p}(0, T ; X)$ the Banach space of all measurable functions $u:] 0, T\left[\rightarrow X\right.$ such that $t \mapsto|u(t)|_{X}$ is in $L^{p}(0, T)$, with norm

$$
|u|_{L^{p}(0, T: X)}=\left(\int_{0}^{T}|u(t)|_{X}^{p} d t\right)^{1 / p},
$$

if $1 \leq p<\infty$, and if $p=\infty$, then

$$
|u|_{L^{p}(0, T ; X)}=\operatorname{ess} \sup |u(t)|_{X} .
$$

We use the following well-known lemmas without the proof in this paper:

Lemma 2.1. (Sobolev - Poincaré) If $u \in H_{0}^{1}(\Omega)$ then $u \in L^{q}(\Omega)$ and the inequality

$$
|u|_{q} \leq C_{q}|\nabla u|
$$

holds, where $q$ is a number satisfying $1 \leq q \leq \frac{2 N}{N-2}$ if $N>2$ and $1 \leq q<\infty$ if $N=2$ and $1 \leq q \leq \infty$ if $N=1$.

Lemma 2.2. (Nakao [6]) Let $\phi(t)$ be a nonnegative bounded function on $[0, \infty[$ satisfying

$$
\sup _{t \leq s \leq t+1} \phi(t)^{1+r} \leq k_{0}(\phi(t)-\phi(t+1))
$$

for $r>0$ and $k_{0}>0$. Then

$$
\phi(t) \leq C(1+t)^{-\frac{1}{r}}, \quad \text { for all } t \geq 0
$$

where $C>0$ is a positive constant depending on $\phi(0)$ and other known constans. 


\section{THE MAIN RESULT}

Theorem 3.1. Let $g: \mathbb{R} \rightarrow \mathbb{R}$ be a non-decreasing continous function such that

$$
\begin{gathered}
g(0)=0 \\
g^{\prime}(s) \geq \tau>0 \\
|g(s)| \leq C_{0}|s|^{q}
\end{gathered}
$$

$C_{0}$ and $\tau$ are two positive constans and $q>1$ is such that $(N-2) q \leq$ $N+2$.

The function a satisfies

$$
\begin{gathered}
a \in W^{1, \infty}(\Omega) \\
a(x) \geq a_{0}>0, \quad \forall x \in \Omega
\end{gathered}
$$

Let. $u_{0} \in H_{0}^{1}(\Omega) \cap H^{2}(\Omega)$ with $u_{0}(x) \neq 0, \forall x \in \Omega$ and $u_{1} \in H_{0}^{1}(\Omega) \cap$ $L^{2 q}(\Omega)$, then exist $\epsilon_{0}>0$ with the following property:

For each $\left\{u_{0}, u_{1}\right\}$ satisfying

$$
2\left(\frac{\left|\nabla u_{1}\right|^{2}}{\left|\nabla u_{0}\right|^{2}}+\left|\triangle u_{0}\right|^{2}\right)<\epsilon_{0}
$$

then exists only one solution $u: \Omega \times] 0, T[\rightarrow \mathbb{R}$ such that

$$
\begin{gathered}
u \in L^{\infty}\left(0, T ; H_{0}^{1} \cap H^{2}\right) \\
u^{\prime} \in L^{\infty}\left(0, T ; H_{0}^{1}\right) \\
u^{\prime \prime} \in L^{\infty}\left(0, T ; L^{2}\right) \\
\frac{d}{d t}\left(u^{\prime}(t), w\right)-|\nabla u(t)|^{2}(\triangle u(t), w)+\left(a(x) g\left(u^{\prime}\right), w\right)=0,
\end{gathered}
$$

$\forall w \in H_{0}^{1}(\Omega)$, in the sense of $D^{\prime}(0, T)$

$$
\begin{gathered}
u(0)=u_{0}, \quad u^{\prime}(0)=u_{1} \\
|\nabla u(t)|>0, \quad \forall t \in[0,+, \infty[
\end{gathered}
$$

Proof. We will use the Faedo-Galerkin method's. 
We consider $\left\{w_{j}\right\}_{j \in \mathbb{N}}$ an orthonormal basis of $H_{0}^{1}(\Omega) \cap H^{2}(\Omega)$ and denote by $V_{m}=\left[w_{1}, \ldots, w_{m}\right]$ the subspace of $H_{0}^{1}(\Omega) \cap H^{2}(\Omega)$ spanned by the first vectors of $\left\{w_{j}\right\}_{j \in \mathbb{N}}$.

We seek $u_{m}(t)$ in the form

$$
u_{m}(t)=\sum_{j=1}^{m} g_{j m}(t) w_{j}
$$

such that, for all $w$ in $V_{m}, u_{m}(t)$ satisfies the approximate equation

$$
\begin{aligned}
\left(u_{m}^{\prime \prime}(t), w\right) & -\left(m^{-1}+\left|\nabla u_{m}(t)\right|^{2}\right)\left(\triangle u_{m}(t), w\right) \\
& +\left(a(x) g\left(u_{m}^{\prime}(t)\right), w\right)=0
\end{aligned}
$$

with the following initial conditions

$$
\begin{aligned}
& u_{m}(0)=u_{0, m} \rightarrow u_{0} \text { in } H_{0}^{1}(\Omega) \cap H^{2}(\Omega) \\
& u_{m}^{\prime}(0)=u_{1, m} \rightarrow u_{1} \text { in } H_{0}^{1}(\Omega) \cap L^{2 q}(\Omega)
\end{aligned}
$$

Using (3.3) we deduce from (3.25) that $\left(g\left(u_{1 m}\right)\right)$ is bounded in $L^{2}(\Omega)$.

Under these conditions, the system (3.13) - (3.15) has a local solution $u_{m}(t)$ over the interval $\left[0, T_{m}\left[\right.\right.$. We shall see that $u_{m}(t)$ can be extended for all $t \geq 0$.

A priori Estimative I

For $w=2 u_{m}^{\prime}(t)$ in (3.13) we find

$$
\begin{gathered}
\frac{d}{d t}\left\{\left|u_{m}^{\prime}(t)\right|^{2}+m^{-1}\left|\nabla u_{m}(t)\right|^{2}+\frac{1}{2}\left|\nabla u_{m}(t)\right|^{4}\right\}+ \\
+2 \int_{\Omega} a(x) g\left(u_{m}^{\prime}(t)\right) u_{m}^{\prime}(t) d x=0
\end{gathered}
$$

Integrate in $[0, t], t<T_{m}$, to obtain

$$
\begin{aligned}
\left|u_{m}^{\prime}(t)\right|^{2}+\frac{1}{2}\left|\nabla u_{m}(t)\right|^{4} & +2 \int_{0}^{t} \int_{\Omega} a(x) g\left(u_{m}^{\prime}\right) u_{m}^{\prime} d x d s \leq \\
& \leq\left|u_{1}\right|^{2}+\left|\nabla u_{0}\right|^{2}+\left|\nabla u_{0}\right|^{4}(3.16)
\end{aligned}
$$

It follows that

$$
\begin{gathered}
\left|u_{m}^{\prime}(t)\right| \leq k \\
\left|\nabla u_{m}(t)\right| \leq k
\end{gathered}
$$


Then we extend the approximate solution $u_{m}(t)$ to the interval $[0, T]$ for any $0<T<\infty$.

From now on we denote by $C$ various constants independent of $m$. and $t$ in $[0, T[$.

Also it follows from (3.16), (3.3) and (3.5) that

$$
\begin{gathered}
\int_{0}^{t} \int_{\Omega} a(x) g\left(u_{m}^{\prime}\right) u_{m}^{\prime} d x d t \leq C \\
\int_{0}^{t} \int_{\Omega}\left|q\left(u_{m}^{\prime}\right)\right|^{\frac{q+1}{q}} d x d t \leq C
\end{gathered}
$$

A priori Estimative II

Putting $w=-2 \triangle u_{m}^{\prime}(t)$ in (3.13) we have

$$
\begin{gathered}
\frac{d}{d t}\left\{\left|\nabla u_{m}^{\prime}(t)\right|^{2}+\left(m^{-1}+\left|\nabla u_{m}(t)\right|^{2}\right)\left|\Delta u_{m}(t)\right|^{2}\right\}+ \\
\left.+2\left(\nabla\left(a(x) g\left(u_{m}^{\prime}\right)\right), \nabla u_{m}^{\prime}\right)\right)=\left(\frac{d}{d t}\left|\nabla u_{m}(t)\right|^{2}\right)\left|\Delta u_{m}(t)\right|^{2}
\end{gathered}
$$

Let us define

$$
F_{m}(t)=\frac{\left|\nabla u_{m}^{\prime}(t)\right|^{2}}{m^{-1}+\left|\nabla u_{m}(t)\right|^{2}}+\left|\triangle u_{m}(t)\right|^{2}=f_{m}(t)+\left|\triangle u_{m}(t)\right|^{2}
$$

A simple computation shows that

$$
\begin{aligned}
F_{m}^{\prime}(t) & =\frac{-2\left(a(x) \nabla g\left(u_{m}^{\prime}\right), \nabla u_{m}^{\prime}\right)-\left(g\left(u_{m}^{\prime}\right) \nabla a(x), \nabla u_{m}^{\prime}\right)}{m^{-1}+\left|\nabla u_{m}(t)\right|^{2}}- \\
& -\frac{2\left(\nabla u_{m}(t), \nabla u_{m}^{\prime}(t)\right)\left|\nabla u_{m}^{\prime}(t)\right|^{2}}{\left(m^{-1}+\left|\nabla u_{m}(t)\right|^{2}\right)^{2}}
\end{aligned}
$$

But:

$$
\left(a \nabla g\left(u^{\prime}\right), \nabla u^{\prime}\right)=\left(a g^{\prime}\left(u^{\prime}\right) \nabla u^{\prime}, \nabla u^{\prime}\right) \geq \tau a_{0}\left|\nabla u^{\prime}\right|^{2}
$$

and using (3.3), the Sobolev embedding and (3.4) give

$$
\left|\left(g\left(u^{\prime}\right) \nabla a, \nabla u^{\prime}\right)\right| \leq C_{0} C_{2 q}|a|_{1, \infty}\left|\nabla u^{\prime}\right|^{q+1}
$$

From (3.22), (3.21), in (3.20) we get 


$$
\begin{aligned}
F_{m}^{\prime}(t) & \leq-2 \tau a_{0} \frac{\left|\nabla u_{m}^{\prime}\right|^{2}}{m^{-1}+\left|\nabla u_{m}\right|^{2}}+2 C_{0} C_{2 q}|a|_{1, \infty} \frac{\left|\nabla u_{m}^{\prime}\right|^{q+1}}{m^{-1}+\left|\nabla u_{m}\right|^{2}} \\
& +2\left[\frac{\left|\nabla u_{m}^{\prime}\right|^{2}}{m^{-1}+\left|\nabla u_{m}\right|^{2}}\right]^{3 / 2}
\end{aligned}
$$

That is:

$$
F_{m}^{\prime}(t) \leq 2\left[f_{m}^{1 / 2}(t)+\delta_{0} f_{m}^{\frac{q-1}{2}}-\delta\right] f_{m}(t)
$$

where $\delta_{0}=C_{0} C_{2 q}|a|_{1, \infty}(1+k)^{\frac{q-1}{2}}, \delta=\tau a_{0}$.

Integrating (3.24) from 0 to $t$ we have

$$
F_{m}(t) \leq 2 \int_{0}^{t}\left(f_{m}^{1 / 2}(s)+\delta_{0} f_{m}^{\frac{q-1}{2}}(s)-\delta\right) f_{m}(s) d s+F_{m}(0)
$$

Now, since $F_{m}(0) \rightarrow F(0)$, it follows of (3.6) that

$$
F_{m}(0)<\epsilon_{0}
$$

for sufficiently large $m$.

We shall prove that

$$
f_{m}^{1 / 2}(t)+\delta_{0} f_{m}^{\frac{q-1}{2}}(t)<\frac{\delta}{2}, \quad \forall t \in[0, \infty[
$$

for $\epsilon_{0}=\min \left\{\left(\frac{\delta}{4}\right)^{2},\left(\frac{\delta}{4 \delta_{0}}\right)^{\frac{2}{q-1}}\right\}$. In fact

$$
\begin{gathered}
f_{m}^{1 / 2}(0) \leq F_{m}^{1 / 2}(0)<\epsilon_{0}^{1 / 2} \leq \frac{\delta}{4} \\
\delta_{0} f_{m}^{\frac{q-1}{2}}(0) \leq \delta_{0} F_{m}^{\frac{q-1}{2}}(0)<\delta_{0} \epsilon_{0}^{\frac{q-1}{2}} \leq \frac{\delta}{4}
\end{gathered}
$$

and thus we have

$$
f_{m}^{1 / 2}(0)+\delta_{0} f_{m}^{\frac{q-1}{2}}(0)<\frac{\delta}{2}
$$

Suppose, then, that (3.27) does not hold for all $t \geq 0$.

Because of the continuity of $f_{m}(t)$, there is $t^{*}>0$ such that

$$
f_{m}^{1 / 2}(t)+\delta_{0} f_{m}^{\frac{q-1}{2}}(t)<\frac{\delta}{2} \quad \text { for } \quad 0 \leq t<t^{*}
$$




$$
f_{m}^{1 / 2}\left(t^{*}\right)+\delta_{0} f_{m}^{\frac{q-1}{2}}\left(t^{*}\right)=\frac{\delta}{2}
$$

(3.25), (3.26) and (3.28) gives:

$$
F_{m}\left(t^{*}\right) \leq F_{m}(0)<\epsilon_{0}
$$

This inequality yields

$$
f_{m}^{1 / 2}\left(t^{*}\right)+\delta_{0} f_{m}^{\frac{q-1}{2}}\left(t^{*}\right)<\frac{\delta}{2}
$$

a contradiction to (3.19). Hence (3.27) is true.

From (3.25) and (3.17) we obtain

wich implies

$$
F_{m}(t)+\delta \int_{0}^{t} \frac{\left|\nabla u_{m}^{\prime}(s)\right|^{2}}{m^{-1}+\left|\nabla u_{m}(s)\right|^{2}} d s \leq C
$$

$$
\begin{gathered}
\left|\nabla u_{m}(t)\right| \leq C \\
\frac{\left|\nabla u_{m}^{\prime}(t)\right|^{2}}{m^{-1}+\left|\nabla u_{m}(t)\right|^{2}} \leq C \\
\int_{0}^{t} \frac{\left|\nabla u_{m}^{\prime}(s)\right|^{2}}{m^{-1}+\left|\nabla u_{m}(s)\right|^{2}} d s \leq C
\end{gathered}
$$

A priori Estimative III

Taking $w=u_{m}^{\prime \prime}(t)$ in (3.13) and choosing $t=0$ we get

$$
\left|u_{m}^{\prime \prime}(0)\right| \leq\left(\frac{1}{m}+\left|\nabla u_{0 m}\right|^{2}\right)\left|\triangle u_{0 m}\right|+|a|_{1, \infty}\left|g\left(u_{1 m}\right)\right|
$$

hence $u_{m}^{\prime \prime}(0)$ is bounded in $L^{2}(\Omega)$. Next, by differentiation of (3.13) and putting $w=2 u_{m}^{\prime \prime}(t)$ we find

$$
\begin{gathered}
\frac{d}{d t}\left\{\left|u_{m}^{\prime \prime}(t)\right|^{2}+\left(m^{-1}+\left|\nabla u_{m}(t)\right|^{2}\right)\left|\nabla u_{m}^{\prime}(t)\right|^{2}\right\}+2 \int_{\Omega} a(x) g^{\prime}\left(u_{m}^{\prime}\right)\left(u_{m}^{\prime \prime}\right) d x \\
=2\left(\nabla u_{m}, \nabla u_{m}^{\prime}\right)\left|\nabla u_{m}^{\prime}(t)\right|^{2}+4\left(\nabla u_{m}, \nabla u_{m}^{\prime}\right) \int_{\Omega}\left(\Delta u_{m}\right) u_{m}^{\prime \prime} d x \\
\leq\left|\nabla u_{m}\right|\left|\nabla u_{m}^{\prime}\right|^{3}+4\left|\nabla u_{m}\right|\left|\nabla u_{m}^{\prime}\right|\left|u_{m}^{\prime \prime}\right|\left|\Delta u_{m}\right| \leq C+C\left|u_{m}^{\prime \prime}(t)\right|^{2}
\end{gathered}
$$

where we have used the priori estimatives I and II.

Integrating from 0 a $t$ we have, using the Gronwall's Inequality: 


$$
\left|u_{m}^{\prime \prime}(t)\right| \leq C
$$

Passage to the limit.

The proof is essentially included in [8]. For completeness however we shall see the convergence of dissipative term. By applying the DunfordPettis and Banach-Bourbaki theorems we conclude from (3.17) - (3.19), (3.30) - (3.32) and (3.33), replacing the sequence $u_{m}$ with a subsequence if needed, that

$$
\begin{array}{ll}
u_{m} \rightarrow u & \text { weak-star in } L^{\infty}\left(0, T ; H_{0}^{1} \cap H^{2}\right) \\
u_{m}^{\prime} \rightarrow u^{\prime} & \text { weak-star in } L^{\infty}\left(0, T ; H_{0}^{1}\right) \\
u_{m}^{\prime \prime} \rightarrow u^{\prime \prime} & \text { weak-star in } L^{\infty}\left(0, T ; L^{2}\right) \\
u_{m}^{\prime} \rightarrow u^{\prime} & \text { almost every where in } Q \\
g\left(u_{m}^{\prime}\right) \rightarrow \chi & \text { weak in } L^{\frac{q+1}{q}}(\Omega)
\end{array}
$$

$$
\left|\nabla u_{m}\right|^{2} \triangle u_{m} \rightarrow \psi \quad \text { weak-star in } \quad L^{\infty}\left(0, T ; L^{2}\right)
$$

We have to show that $u$ is a solution of $(\mathrm{P})$. We shall prove only that

$$
\int_{Q} a(x) g\left(u_{m}^{\prime}\right) v d x d t \rightarrow \int_{Q} a(x) g\left(u^{\prime}\right) v d x d t
$$

for all $v \in L^{q+1}\left(0, T ; H_{0}^{1}(\Omega)\right)$.

In fact, from (3.18) and Fatou's Lemma $u^{\prime} g\left(u^{\prime}\right) \in L^{\prime}(Q)$. This yield $g\left(u^{\prime}\right) \in L^{1}(Q)$. On the other hand (3.37) and the continuity of $g$ we deduce that

$$
g\left(u_{m}^{\prime}\right) \rightarrow g\left(u^{\prime}\right) \quad \text { a.e. in } Q
$$

Let $E \subseteq Q$ and set

$$
E_{1}=\left\{(x, t) \in E: g\left(u_{m}^{\prime}(x, t)\right) \leq|E|^{-1 / 2}\right\} ; \quad E_{2}=E-E_{1}
$$

when $|E|$ is the measure of $E$.

If $h(r)=\inf \{|x|: x \in \mathbb{R}$ and $|g(x)| \geq r\}$ then we have

$$
\int_{E}\left|g\left(u_{m}^{\prime}\right)\right| d x d t \leq|E|^{1 / 2}+\left[h\left(|E|^{-1 / 2}\right)\right]^{-1} \int_{E_{2}}\left|u_{m}^{\prime} g\left(u_{m}^{\prime}\right)\right| d x d t
$$

Applying (3.18) we have that 


$$
\sup _{m \in \mathbb{N}} \int_{E}\left|g\left(u_{m}^{\prime}(x, t)\right)\right| d x d t \rightarrow 0 \quad \text { when } \quad|E| \rightarrow 0 .
$$

From Vitali's convergence theorem we get

Hence we have

$$
g\left(u_{m}^{\prime}\right) \rightarrow g\left(u^{\prime}\right) \quad \text { in } \quad L^{1}(Q)
$$

$$
\int_{0}^{T} \int_{\Omega} a(x)\left[g\left(u_{m}^{\prime}\right)-g\left(u^{\prime}\right)\right] d x d t \leq|a|_{1, \infty}\left|g\left(u_{m}^{\prime}\right)-g\left(u^{\prime}\right)\right|_{L^{\prime}(Q)} \rightarrow 0
$$

as $m \rightarrow \infty$.

So we get that

$$
a(x) g\left(u_{m}^{\prime}\right) \rightarrow a(x) g\left(u^{\prime}\right) \quad \text { in } L^{\prime}(Q)
$$

and from (3.38)

$$
a(x) g\left(u_{m}^{\prime}\right) \rightarrow a(x) g\left(u^{\prime}\right) \quad \text { weak-star in } L^{\frac{q+1}{q}}(Q)
$$

this implies (3.39).

We now prove that $|\nabla u(t)|>0$ for all $t \geq 0$. We need the following lemma

Lemma. If $v:[-T, T] \rightarrow H_{0}^{1}(\Omega) \cap H^{2}(\Omega)$ is a weak solution of

$$
\begin{aligned}
& v^{\prime \prime}(t)-|\nabla v(t)|^{2} \triangle v(t)+a(x) g\left(v^{\prime}(t)\right)=0, \quad-T \leq t \leq T \\
& v(0)=0, \quad v^{\prime}(0)=0
\end{aligned}
$$

then $v(t)=0$, for $t \in[-T, T]$.

Proof. Multiplying with $2 v^{\prime}(t)$ we have

$$
\frac{d}{d t}\left\{\left|v^{\prime}(t)\right|^{2}+\frac{1}{2}|\nabla v(t)|^{4}\right\}+2 \int_{\Omega} a(x) g\left(v^{\prime}(t)\right) v^{\prime}(t) d x=0
$$

and integrating in $[0, t]$, using $(3.2)$, gives

$$
\left|v^{\prime}(t)\right|^{2}+\frac{1}{2}|\nabla v(t)|^{4} \leq 2 a_{0}|\tau| \int_{0}^{|t|}\left|v^{\prime}(s)\right|^{2} d s
$$

Gronwall's Lemma assures $v^{\prime}(t)=0$ and $v(t)=0$ for all $t \in[-T . T]$. This concludes the proof of this lemma. 
We now turn to the proof of $|\nabla u(t)|>0, \quad \forall t \geq 0$. Suppose that there exists a number $T>0$ such that $\nabla u(T)=0$. Since the a priori estimatives imply that $\frac{\left|\nabla u^{\prime}(T)\right|}{|\nabla u(T)|}$ is bounded, then

$$
\left|\nabla u^{\prime}(T)\right| \leq C|\nabla u(T)|=0 .
$$

Hence, the above lemma implies that $u(t)=0$, for $0 \leq t \leq T$, wich contradicts $u_{0}(x) \neq 0$.

The uniqueness is a consequence of the monotonicity of $g$ and Gronwall's Inequality. We shall omit the proof. Since it can be obtained in a standard way.

Theorem 3.2. (Energy Decay) In addition to (3.1) - (3.3), assume that

$$
g(s) \leq C_{1}|s| \text { if }|s| \leq 1
$$

then the total energy

$$
E(t)=\left|u^{\prime}(t)\right|^{2}+\frac{1}{2}|\nabla u(t)|^{4}
$$

satisfies

$$
E(t) \leq \frac{C_{2}}{(1+t)^{2}}, \quad \text { for all } t \geq 0
$$

where $C_{1}$ and $C_{2}$ are positive constants.

Proof. Taking the scalar product of the first equation of $(P)$ with $2 u^{\prime}$ and integrating over $\Omega$ we obtain

$$
E^{\prime}(t)+2 \int_{\Omega} a(x) u^{\prime} g\left(u^{\prime}\right) d x=0
$$

Integrating (3.41) over $[t, t+1]$ we get

$$
2 \int_{t}^{t+1} \int_{\Omega} a(x) u^{\prime} g\left(u^{\prime}\right) d x d s=E(t)-E(t+1) \equiv D(t)^{2}
$$

From this we obtain

$$
\int_{t}^{t+1}\left|u^{\prime}(s)\right|^{2} d s \leq C D(t)^{2}
$$


By the Mean Value theorem there exist two points $t_{1} \in\left[t, t+\frac{1}{4}\right]$ and $t_{2} \in\left[t+\frac{3}{4}, t+1\right]$ such that

$$
\left|u^{\prime}\left(t_{i}\right)\right|^{2} \leq C D(t)^{2}, \quad i=1,2
$$

thus, multiplying of the first equation in $(P)$ by $u$ and integrating it over $\Omega \times] t_{1}, t_{2}$ [, we have from (3.42) - (3.44) and Lemma 2.1

$$
\begin{aligned}
\int_{t_{1}}^{t_{2}}|\nabla u(s)|^{4} d s= & \int_{t_{1}}^{t_{2}}\left|u^{\prime}(s)\right|^{2} d s-\left(u^{\prime}\left(t_{1}\right), u\left(t_{1}\right)\right)+\left(u^{\prime}\left(t_{2}\right), u\left(t_{2}\right)\right) \\
& -\int_{t_{1}}^{t_{2}}\left(a(x) g\left(u^{\prime}\right), u\right) d s \\
\leq & C_{(}\left(D(t)^{2}+D(t) E(t)^{1 / 4}\right) \equiv A(t)^{2}
\end{aligned}
$$

From (3.43) and (3.45) we conclude that,

$$
E\left(t_{2}\right) \leq \frac{1}{t_{2}-t_{1}} \int_{t_{1}}^{t_{2}} E(s) d s \leq C A(t)^{2}
$$

there fore, we obtain

$$
\begin{aligned}
E(t) & =E\left(t_{2}\right)+2 \int_{t}^{t_{2}} \int_{\Omega} a(x) g\left(u^{\prime}\right) u^{\prime} d x d s \\
& \leq C A(t) \leq C\left\{D(t)^{2}+D(t) E(t)^{1 / 4}\right\}
\end{aligned}
$$

Using Young's inequality, noting that $D(t)^{2} \leq E(t) \leq E(0)$ we get

$$
\sup _{t \leq s \leq t+1} E(s)^{3 / 2} \leq C D(t)^{2}=C(E(t)-E(t+1))
$$

Hence, lemma 2.2 gives

$$
E(t) \leq C(1+t)^{-2}, \quad \forall t \geq 0
$$

this ends the proof of theorem. 


\section{REFERENCES}

[1] M. Aassila, Global Existence of Solutions to degenerate wave equations uith dissipalive terms, Bull. Austral. Mat.h. Soc. (6) (1999), p. 1 - 10.

[2] E. Cabanillas, Existencia Global y Comportamiento Asintótico para una ecuación de Kirchoff con término disipativo de Coeficiente Variable, Pesquimat, Revista de Investigación de la FCM - UNMSM Vol.III, №2 (2000) p.44 - 50. LIMA - PERU.

[3] L. A. Medeiros, M. Milla Miranda, Espaços de Sobolev - Iniciação aos Problemas elípticos não Homogéneos, Instituto de' Matemática, UFRJ, Río de Janeiro, First edition, 1997.

[4] T. Mizumachi, Time decay of solutions to degenerate Kirchoff type equation, preprint (1997).

[5] K. Mochizuki, Global existence and energy decay of small solutions to the Kirchoff equation with linear dissipation localized near infinity, J. Math. Kyoto Univ. (JMKYAZ) 39 - 2 (1999) p. $347-363$.

[6] M. Nakao, A Difference Inequality and its application to nonlinear evolution equations, J. Math. Soc. Japan 30 (1978) p. 747 - 762.

[7]'K. Ono, On Global existence and Asymplotic stability of solutions of midly degenerate dissipative wave equations of Kirchoff Type, Asymplotic: Analysis 16 (1998) p. 299 - 314.

[8] S. K. Patcheu, Global Existence and Exponential decay estimatives for a damped quasilinear equation, Comm. Part. Difr. Eq. 22 (11 \& 12) (1997) p. $2007-2(024$. 